\title{
POSITIVE TEMPERATURES ON AN INFINITE ROD
}

\author{
BY \\ D. V. WIDDER
}

1. Introduction. There is a familiar integral representation of the temperatures on an infinite insulated rod. It is usually known by the name of Poisson $(1835 ; 140)\left({ }^{1}\right)$ though it was known to Fourier $(1822 ; 454)$ and to Laplace $(1809 ; 241)$. It was later used by Weierstrass (1885) to give the first proof that an arbitrary continuous function can be uniformly approximated by polynomials. There is a question of uniqueness, however, which was not discussed by any of these authors, nor has it been completely answered to this day. Very important information on the question has been discovered in recent years, notably by Tychonoff and Täcklind. These results are described below.

The Poisson representation is in terms of the so-called "source solution" or "fundamental solution"

$$
k(x, t)=(4 \pi t)^{-1 / 2} e^{-x^{2} / 4 t}
$$

of the heat equation

$$
\partial^{2} u / \partial x^{2}=\partial u / \partial t
$$

It is

$$
u(x, t)=\int_{-\infty}^{\infty} k(y-x, t) \phi(y) d y .
$$

Here $u(x, t)$ is the temperature of an infinite insulated rod, which we think of as extended along the $x$-axis of an $x t$-plane, at the point $x$ of the rod and at the time $t$. The function $\phi(y)$ is the temperature of the rod at the point $x=y$ at the initial time $t=0$. It is easy to believe that the function $u(x, t)$ satisfies equation (2), since the function $k(x, t)$ itself does. It is further likely that $u(x, t)$ tends to $\phi(x)$ as $t$ tends to zero through positive values. This becomes intuitively obvious when one observes that for any fixed positive $t$ the area under the curve $y=k(x, t)$ is unity, that the curve has a unique maximum at $x=0$ and that $k(x, t)$ tends to zero with $t$ for each $x$ different from zero. From these facts it follows that $k(0, t)$ becomes positively infinite as $t$ approaches zero. Hence for very small positive values of $t$ the integral (3) is approximately equal to the value of $\phi(y)$ at the point $y=x$ where the kernel $k(y-x, t)$ has its maximum value.

Presented to the Society, September 13, 1943; received by the editors September 7, 1943.

(1) The dates in parentheses refer to the bibliography at the end of this paper. A number following the date indicates the page number of the article to which reference is made. 
As we mentioned above, the problem of the uniqueness of this classic solution has not been completely solved. It is easy to see that it is impossible to impose uniqueness conditions on the function $\phi(y)$ alone. For, the function $v(x, t)=(x / t) k(x, t)$ is a solution of equation (2) which approaches zero with $t$ for every fixed $x$. Hence the functions $u(x, t)+c v(x, t)$ are solutions of the original boundary value problem for all constants $c$.

However, certain authors have been able to impose uniqueness conditions on the function $u(x, t)$ with respect to its behavior in portions of the $x t$-plane near $x= \pm \infty$. For a summary of results in this direction obtained prior to 1936, see Doetsch (1936). There appears an extensive bibliography on the subject. One useful source not there mentioned is Goursat $(1923 ; 311)$. Goursat proves that if the functions $u(x, t) e^{-K x^{2}}$ and $(\partial u / \partial x) e^{-K x^{2}}$ are bounded in the half-plane $t>0$ for some positive constant $K$, then the function (3) provides the unique solution of the problem. Titchmarsh $(1937 ; 282)$ replaces these two conditions by the single one that the function $u(x, t) e^{-K|x|}$ is bounded. Tychonoff $(1935 ; 199)$ shows that the exponent $|x|$ can be replaced by $x^{2}$ but by no higher power of $|x|$. Täckland (1936) replaces the factor $e^{-K|x|}$ by $e^{-|x| h(|x|)}$ where the function $h(x)$ belongs to a quasi-analytic class of functions of Denjoy and Carleman. Thus, in particular, $h(x)$ could be any positive function which makes the integral $\int^{\infty} d x / h(x)$ diverge; for example, the function $x \log x$.

In the present paper a uniqueness condition of another type is obtained. It is shown that if the temperature is always positive for positive time, then it cannot be identically zero at time zero. The proof is based on the work of Tychonoff and Täcklind. Since each of the papers in which these results are obtained is highly inaccessible, the second section of the present paper is devoted to just that part of their results which we need. The paper is thus complete in itself. It is the more elegant approach of Tychonoff which we employ.

The original purpose of the present paper was to establish a Stieltjes integral representation of the type (3) for solutions of equation (2) which are positive for positive time. This would be analogous to the theorem of Herglotz $(1911 ; 501)$ for a Stieltjes integral representation of positive functions harmonic in a circle or a half-plane. (For a recent proof see Loomis and Widder $(1942 ; 643)$.) The establishment of the uniqueness theorem required for the representation result turned out to be the major difficulty. We prove that a solution of equation (2) is non-negative for $t>0$ if and only if it has the Poisson-Stieltjes representation

$$
u(x, t)=\int_{-\infty}^{\infty} k(y-x, t) d \alpha(y),
$$

where $\alpha(y)$ is a non-decreasing function.

It is known that any solution $u(x, t)$ of the heat equation (2) is necessarily 
an analytic function of $x$. As a function of $t$, however, the solution need not be analytic $\left({ }^{2}\right)$. But we now see that any solution which is non-negative in a half-plane (or strip) must be analytic in each of the variables $x$ and $t$. This follows easily from the integral representation. We have here one more instance in which the positiveness of an operator is associated with analytic$\operatorname{ity}\left({ }^{3}\right)$.

2. The work of Tychonoff. We shall denote the set of points $(x, t)$ for which $-R<x<R, 0<t \leqq c$ by $D_{R, c}$. This is a rectangle without three of its boundary lines. The set of points on these three boundary lines will be denoted by $B_{R, c}$. If the dimensions of the rectangle need not be put into evidence we shall drop the subscripts. Thus the set of points $D+B$ is a closed rectangle.

A function $u(x, t)$ which is of class $C^{2}$ and satisfies the heat equation (2) will be said to belong to the class $H$. The proof of our first result follows essentially an outline given in a footnote of the paper of Tychonoff (1935). The result was known to many earlier writers.

TheOREM A. If

1. $u(x, t) \in H$ in $D$,

2. $\lim \inf _{x \rightarrow x_{0}, t \rightarrow t_{0}} u(x, t) \geqq 0$ for all $\left(x_{0}, t_{0}\right)$ on $B$, then

$$
u(x, t) \geqq 0 \text { in } D .
$$

By hypothesis 2 it follows that for each $\left(x_{0}, t_{0}\right)$ of $B$ and for each $\epsilon>0$ there corresponds a $\delta_{0}>0$ such that $u(x, t)>-\epsilon$ for all points $(x, t)$ of $D$ within a distance $\delta_{0}$ of $\left(x_{0}, t_{0}\right)$. It follows by an obvious use of the HeineBorel theorem that to an arbitrary $\epsilon>0$ there corresponds a $\delta>0$ such that $u(x, t)>-\epsilon$ at all points $(x, t)$ of $D$ within a distance $\delta$ of $B$.

Now suppose there existed a point $\left(x_{1}, t_{1}\right)$ of $D$ where $u\left(x_{1}, t_{1}\right)=-l<0$, contrary to inequality (4). Form the function

$$
v(x, t)=u(x, t)+k t-k t_{1}
$$

where $0<k<l / t_{1}$. Now choose $\epsilon>0$ such that $k t_{1}+\epsilon<l$, and determine the $\delta$ which corresponds to it according to the above remark. Then for points of $D$ within a distance $\delta$ of $B$ we have $v(x, t)>-l$. Since $v\left(x_{1}, t_{1}\right)=-l$ it is clear that the minimum of $v(x, t)$ is attained at a point $\left(x_{2}, t_{2}\right)$ of $D$. But this will contradict hypothesis 1 . For, in terms of $v(x, t)$ equation (2) becomes

$$
\partial^{2} v / \partial x^{2}=\partial v / \partial t-k .
$$

(2) Examples may be found in the article of Tychonoff (1939).

(3) Compare the inversion operator $\lim _{k \rightarrow \infty}(-1)^{k} f^{(k)}(k / t)(k / t)^{k+1}$ of the Laplace integral. From the positiveness of the operator, we infer the theorem of S. Bernstein that $(-1)^{k} f^{(k)}(x) \geqq 0$ for $x>0$ and for $k=0,1,2, \cdots$ implies the analyticity of $f(x)$. In the present case one inverts the integral (3) simply by the formula $\lim _{t \rightarrow 0+} u(x, t)$. Consequently it should not be surprising that the positiveness of $u(x, t)$ near the $x$-axis should bring with it the analyticity of $u(x, t)$. 
But at a minimum $\partial^{2} v / \partial x^{2} \geqq 0$ and $\partial v / \partial t \leqq 0$ (the inequality being possible if $t_{2}=c$ ), and these inequalities are untenable by virtue of equation (5). Hence the assumption that $u(x, t)$ was negative at a point of $D$ must have been false, and the theorem is proved.

The next result is the main uniqueness result of Tychonoff.

THEOREM B. If

1. $u(x, t) \in H$ in the strip $0<t \leqq c$,

2. $\lim _{x \rightarrow x_{0}, t \rightarrow 0+} u(x, t)=0$ for all $x_{0}$,

3. $f(x)=\max _{0<t \leqq c}|u(x, t)|$,

4. $f(x)=O\left(e^{a x^{2}}\right)(x \rightarrow \pm \infty)$ for some $a>0$, then $u(x, t)=0$ throughout the strip $0<t \leqq c$.

Form the auxiliary function $U_{R}(x, t)=f(-R) k(x+R, t)+f(R) k(x-R, t)$, $R>0$. Then $U_{R}(x, t) \in H$ and $U_{R}( \pm R, t) \geqq f( \pm R) k(0, t)=f( \pm R)(4 \pi t)^{-1 / 2}$, $t>0$. Hence from the definition of $f(x)$ we have, for $0<t \leqq c$,

$$
|u( \pm R, t)| \leqq f( \pm R) \leqq f( \pm R)(c / t)^{1 / 2} \leqq(4 \pi c)^{1 / 2} U_{R}( \pm R, t) .
$$

That is, the functions $(4 \pi c)^{1 / 2} U_{R}(x, t) \pm u(x, t)$ both belong to $H$ in $D_{R, c}$ and are non-negative on $B_{R, c}$. On the lower boundary they both vanish except at the end points $( \pm R, 0)$, where they both have a non-negative limit inferior. Hence by Theorem A $|u(x, t)| \leqq(4 \pi c)^{1 / 2} U_{R}(x, t)$ throughout $D_{R, c}$. Now hold $(x, t)$ fixed and let $R$ become infinite. The function $U_{R}(x, t)$ tends to zero if $0<t<1 /(4 a)$. If $1 /(4 a) \geqq c$ the proof is complete. Otherwise repeat the above argument with $u(x, t)$ replaced by $u\left(x, t+(4 a)^{-1}\right)$, and so forth. Observe that hypothesis 2 may not be replaced by $u(x, 0+)=0$ for all $x$. The example $x t^{-1} k(x, t)$ shows this.

3. Convergence and inversion of the Poisson integral. In this section we prove that the Poisson integral (3) converges and represents a function of $H$ under suitable restrictions on $\phi(y)$, and that this function tends to $\phi(y)$ as the point $(x, t)$ tends to the point $(y, 0)$. The results are of course known, but it is impossible to give a reference to them in the precise form needed for our subsequent work.

TheOREM 1. If

1. $\phi(y) e^{-a y^{2}} \in L(-\infty, \infty)$ for some $a>0$,

2. $F(x, t)=\int_{-\infty}^{\infty} k(x-y, t) \phi(y) d y$, then $F(x, t)$ is defined and belongs to $H$ in the strip $0<t<1 /(4 a)$.

Formal differentiation gives

$$
\begin{gathered}
\partial^{2} F / \partial x^{2}=\partial F / \partial t=\frac{1}{4 t^{2}} \int_{-\infty}^{\infty}(y-x)^{2} k(y-x, t) \phi(y) d y \\
-\frac{1}{2 t} \int_{-\infty}^{\infty} k(y-x, t) \phi(y) d y .
\end{gathered}
$$


Our result will be established if we can show that both integrals converge uniformly in some neighborhood of an arbitrary point $\left(x_{0}, t_{0}\right)$ of the strip $0<t<1 /(4 a)$. Choose $\delta$ so small that the square $\left|x-x_{0}\right|<\delta,\left|t-t_{0}\right|<\delta$ lies in the strip. It will be sufficient to show that both integrals are dominated by convergent integrals whose integrands are independent of $(x, t)$ in the square. Consider separately the pair of integrals over the partial range $R$ to $\infty$ $\left(R>x_{0}+\delta\right)$ and the pair over the range $-\infty$ to $S\left(S<x_{0}-\delta\right)$. To treat the first pair we have for $(x, t)$ in the square and for $y \geqq R$

$$
\begin{aligned}
k(y-x, t) & <C k\left(y-x_{0}-\delta, t_{0}+\delta\right), \\
(y-x)^{2} k(y-x, t) & <C\left(y-x_{0}+\delta\right)^{2} k\left(y-x_{0}-\delta, t_{0}+\delta\right), \\
C & =\left(t_{0}+\delta\right)^{1 / 2}\left(t_{0}-\delta\right)^{-1 / 2} .
\end{aligned}
$$

Since $t_{0}+\delta<1 /(4 a)$, it is clear that the functions $k\left(y-x_{0}-\delta, t_{0}+\delta\right) e^{a y^{2}}$ and $\left(y-x_{0}+\delta\right)^{2} k\left(y-x_{0}-\delta, t_{0}+\delta\right) e^{a y^{2}}$ are bounded functions of $y$ in the interval $(-\infty, \infty)$. Hence the two integrals in question are dominated by a constant multiple of the integral

$$
\int_{R}^{\infty} e^{-a y^{2}}|\phi(y)| d y,
$$

which is independent of $x$ and $t$ in the square. The other two integrals are handled in a similar way, so that the proof is complete.

THEOREM 2. If $\phi(y)$ and $F(x, t)$ are defined as in Theorem 1, and if $\phi\left(y_{0}+\right)$ and $\phi\left(y_{0}-\right)$ exist, then

$$
\lim _{x \rightarrow y_{0}, t \rightarrow 0+}|F(x, t)| \leqq M=\max \left(\left|\phi\left(y_{0}+\right)\right|,\left|\phi\left(y_{0}-\right)\right|\right) .
$$

If in addition $\phi\left(y_{0}+\right)=\phi\left(y_{0}-\right)$, then

$$
\lim _{x \rightarrow y_{0}, t \rightarrow 0+} F(x, t)=\phi\left(y_{0}+\right) \text {. }
$$

For, to an arbitrary positive $\epsilon$ there corresponds a $\delta$ such that

Then

$$
|\phi(y)|<M+\epsilon, \quad\left|y-y_{0}\right| \leqq \delta .
$$

$$
F(x, t)=\left\{\int_{-\infty}^{y_{0}-\delta}+\int_{y_{0}-\delta}^{y_{0}+\delta}+\int_{y_{0}+\delta}^{\infty}\right\} k(y-x, t) \phi(y) d y=I_{1}+I_{2}+I_{3} .
$$

Clearly

$$
\left|I_{2}\right| \leqq(M+\epsilon) \int_{-\infty}^{\infty} k(y-x, t) d y=M+\epsilon .
$$

If $\left|x-y_{0}\right|<\rho<\delta$, then 


$$
\left|I_{3}\right| \leqq \int_{y_{0}+\delta}^{\infty} k\left(y-y_{0}-\rho, t\right)|\phi(y)| d y .
$$

Since $k\left(y-y_{0}-\rho, t\right) e^{a y^{2}}$ is a decreasing function of $y$ for $y \geqq y_{0}+\delta$ when $t$ is sufficiently small (it has its maximum at $\left.\left(y_{0}+\rho\right) /(1-4 a t)\right)$, we see that for such $t$

$$
\left|I_{3}\right| \leqq k(\delta-\rho, t) e^{a\left(y_{0}+\delta\right)^{2}} \int_{y_{0}+\delta}^{\infty} e^{-a y^{2}}|\phi(y)| d y .
$$

Thus $I_{3}$ tends to zero with $t$. The integral $I_{1}$ does also. Hence

$$
\lim _{x \rightarrow y_{0}, t \rightarrow 0+}|F(x, t)| \leqq M+\epsilon,
$$

from which inequality (6) follows at once.

To prove equation (7) we express $F(x, t)-\phi\left(y_{0}+\right)$ as the sum of three integrals like those in equation (8) except that $\phi(y)$ is replaced by $\phi(y)$ $-\phi\left(y_{0}+\right)$ in the integrand. As before

$$
\lim _{x \rightarrow y_{0}, t \rightarrow 0+} \sup _{t \rightarrow}\left|F(x, t)-\phi\left(y_{0}+\right)\right| \leqq \operatorname{liu.b.}_{\left|y-y_{0}\right| \leqq \delta}\left|\phi(y)-\phi\left(y_{0}+\right)\right| .
$$

Since the right-hand side approaches zero with $\delta$ the theorem is completely proved.

4. The uniqueness theorem. In this section we prove that there cannot be a positive solution of equation (2) which vanishes for time zero. We need two preliminary results.

TheOREM 3. If

1. $\phi(y) \in L(-A, A)$,

2. $F_{A}(x, t)=\int_{-4}^{A} k(y-x, t) \phi(y) d y, 0<t<\infty$, then

3. $g(x)=$ l.u.b. $0<t \leqq c\left|F_{A}(x, t)\right|$ for some $c>0$,

$$
\lim _{x \rightarrow \pm \infty} g(x)=0 \text {. }
$$

When $|x|>A$ we have

$$
k(y-x, t) \leqq(4 \pi t)^{-1 / 2} e^{-(|x|-A)^{2} / 4 t}, \quad|y| \leqq A .
$$

If $\delta$ is a positive constant it is easy to see that

$$
z e^{-\delta^{2} z^{2}} \leqq(2 e)^{-1 / 2} \delta^{-1}, \quad 0 \leqq z<\infty,
$$

so that

$$
k(y-x, t) \leqq(2 \pi e)^{-1 / 2}(|x|-A)^{-1}, \quad|y| \leqq A .
$$

Hence for $|x|>A$ 


$$
\left|F_{A}(x, t)\right| \leqq(2 \pi e)^{-1 / 2}(|x|-A)^{-1} \int_{-A}^{A}|\phi(y)| d y,
$$

from which the result is immediate.

TheOREM 4. If

1. $u(x, t) \in H, 0<t<c$,

2. $u(x, t) \geqq 0,0<t<c$,

then the integral

$$
\int_{-\infty}^{\infty} k(y-x, t) u(y, \delta) d y
$$

exists for $0<\delta<c,-\infty<x<\infty, 0<t<c-\delta$ and is not greater than $u(x, t+\delta)$.

Consider the function $v(x, t)=u(x, t+\delta)-F_{A}(x, t)$, where $F_{A}(x, t)$ is the function of Theorem 3 with $\phi(y)=u(y, \delta)$. By Theorem $1, v(x, t) \in H$ for $0<t<c-\delta$. By Theorem 2,

so that

$$
\begin{array}{ll}
\lim _{x \rightarrow x_{0}, t \rightarrow 0+} F_{A}(x, t)= \begin{cases}u\left(x_{0}, \delta\right), & \left|x_{0}\right|<A, \\
0, & \left|x_{0}\right|>A,\end{cases} \\
\limsup _{x \rightarrow \pm A, t \rightarrow 0+} F_{A}(x, t) \leqq u( \pm A, \delta), &
\end{array}
$$$$
\lim _{x \rightarrow x_{0}, t \rightarrow 0+} v(x, t) \geqq 0, \quad-\infty<x_{0}<\infty .
$$

We can now conclude that $v(x, t) \geqq 0$ in the strip $0<t<c-\delta$. For suppose $v\left(x_{0}, t_{0}\right)=-l<0$ at some point $\left(x_{0}, t_{0}\right)$ of that strip. By Theorem 3 we can determine $R$ so large that $v(x, t) \geqq-l / 2$ on the line segments $x= \pm R$, $0<t<c-\delta$. Now apply Theorem $A$ to the function $v(x, t)+l / 2$ in the rectangle $D_{R, c^{\prime}}$, where $t_{0}<c^{\prime}<c-\delta$. We conclude that $v(x, t) \geqq-l / 2$ throughout the rectangle. But this is impossible at $\left(x_{0}, t_{0}\right)$. Hence

$$
F_{A}(x, t) \leqq u(x, t+\delta)
$$

in the strip $0<t<c-\delta$. Now allowing $A$ to become infinite we obtain our result, by virtue of hypothesis 2 .

THEOREM 5. If

1. $u(x, t) \in H, 0 \leqq t<c$,

2. $u(x, t) \geqq 0,0 \leqq t<c$,

3. $u(x, 0)=0,-\infty<x<\infty$,

then $u(x, t)$ vanishes identically in the strip $0 \leqq t<c$.

Set

$$
w(x, t)=\int_{0}^{t} u(x, y) d y .
$$


Since $u(x, t) \in C^{2}$ on the $x$-axis by hypothesis 1 , we have

$$
\begin{array}{rlrl}
\left(\partial^{2} / \partial x^{2}\right) w(x, t) & =\int_{0}^{t}\left(\partial^{2} / \partial x^{2}\right) u(x, y) d y & \\
& =\int_{0}^{t}(\partial / \partial y) u(x, y) d y=u(x, t), & & 0 \leqq t<c, \\
(\partial / \partial t) w(x, t) & =u(x, t), & & 0 \leqq t<c .
\end{array}
$$

Consequently, we see that $w(x, t) \in H$ in $0 \leqq t<c$. It satisfies all the hypotheses of the theorem, but has the additional properties of being convex in $x$ and non-decreasing in $t$. Moreover, if $w(x, t)$ vanishes identically, the same is true of $u(x, t)$. Hence, there is no loss in generality if we add the hypotheses of convexity and monotoneity to the original function $u(x, t)$.

Let $\delta$ be an arbitrary positive number less than $c$ and set $x=0, t=t_{0}<c-\delta$ in the integral (9). Then

$$
\int_{-\infty}^{\infty} e^{-y^{2} / 4 t_{0}} u(y, \delta) d y<\infty, \quad 0<\delta<c .
$$

Since $u(x, t)$ is non-negative and non-decreasing in $t$,

$$
f(x)=\max _{0 \leqq t \leqq \delta} u(x, t)=u(x, \delta) .
$$

By the convexity of $f(x)$ we have

$$
2 x f(x) e^{-x^{2} / t_{0}} \leqq e^{-x^{2} / t_{0}} \int_{0}^{2 x} f(y) d y \leqq \int_{0}^{2 x} f(y) e^{-y^{2} / 4 t_{0}} d y \leqq \int_{-\infty}^{\infty} f(y) e^{-y^{2} / 4 t_{0}} d y .
$$

The last integral converges by inequality (10). Hence

$$
f(x)=O\left(e^{x^{2} / t_{0}}\right),
$$$$
x \rightarrow \infty \text {. }
$$

It is easily seen that relation (11) also holds for $x \rightarrow-\infty$. All hypotheses of Theorem $\mathrm{B}$ are satisfied in the strip $0<t \leqq \delta$ so that $u(x, t)$ vanishes there. Since $\delta$ was arbitrary our theorem is proved.

5. The Poisson-Stieltjes integral representation. We now obtain an integral expression for functions of $H$ which are positive in a horizontal strip.

Theorem 6. $A$ necessary and sufficient condition that $u(x, t) \in H$ and $u(x, t) \geqq 0$ in the strip $0<t<c$ is that

$$
u(x, t)=\int_{-\infty}^{\infty} k(y-x, t) d \alpha(y),
$$

where $\alpha(y)$ is non-decreasing and the integral converges in the strip.

The sufficiency of the condition follows if we note that $k(y-x, t)>0$ and 
observe that differentiation under the integral sign with respect to $x$ or $t$ is permissible. The proof of the latter fact is essentially that given in the proof of Theorem 1.

To prove the necessity set

$$
\beta_{\delta}(x)=\int_{-\infty}^{x} k\left(y, t_{0}\right) u(y, \delta) d y,
$$

where $0<\delta<c, 0<t_{0}<c-\delta$. Then by Theorem 4

$$
0 \leqq \beta_{\delta}(x) \leqq u\left(0, t_{0}+\delta\right) \text {. }
$$

Also by Theorem 2

$$
\begin{aligned}
u\left(x_{0}, \delta\right) & =\lim _{x \rightarrow x_{0}, t \rightarrow 0+} \int_{-\infty}^{\infty} \frac{k(y-x, t)}{k\left(y, t_{0}\right)} d \beta_{\delta}(y) \\
& =\lim _{x \rightarrow x_{0}, t \rightarrow 0+} \int_{-\infty}^{\infty} k(y-x, t) u(y, \delta) d y .
\end{aligned}
$$

The function $u(x, t+\delta)$ approaches the same value and by Theorem 4

$$
\int_{-\infty}^{\infty} k(y-x, t) u(y, \delta) d y \leqq u(x, t+\delta)
$$

for $0<t<c-\delta$. Hence by Theorem 5

$$
\begin{aligned}
u(x, t+\delta) & =\int_{-\infty}^{\infty} \frac{k(y-x, t)}{k\left(y, t_{0}\right)} d \dot{\beta}_{\delta}(y), \\
u(x, t) & =\lim _{\delta \rightarrow 0} \int_{-\infty}^{\infty} \frac{k(y-x, t)}{k\left(y, t_{0}\right)} d \beta_{\delta}(y),
\end{aligned}
$$

for $0<t<c$. We may now apply the Helly theorem( $\left.{ }^{4}\right)$ and the Helly-Bray theorem( $\left.{ }^{4}\right)$ in the standard way to obtain

$$
u(x, t)=\int_{-\infty}^{\infty} \frac{k(y-x, t)}{k\left(y, t_{0}\right)} d \beta(y) .
$$

Here $\beta(y)$ is non-decreasing and bounded, being the limit of a sequence of functions taken from the set $\beta_{\delta}(y)$. We thus obtain (12), where

$$
\alpha(y)=\int_{0}^{y} \frac{1}{k\left(x, t_{0}\right)} d \beta(x), \quad-\infty<y<\infty .
$$

This function is clearly non-decreasing, so that the proof is complete.

6. A refinement of the uniqueness theorem. In $\$ 4$ we showed that there

(') See, for example, Widder (1941; 26-32). 
exists no solution of the heat equation which is everywhere zero at time zero and positive thereafter. In that section we understood that $u(x, t)$ was continuous in the neighborhood of the $x$-axis. In fact we even assumed that $u(x, t) \in H$ on that axis.

We can now extend the uniqueness theorem to include the less restrictive boundary condition

$$
u(x, 0+)=0, \quad-\infty<x<\infty .
$$

This is the more desirable type of condition from the physical point of view $\left(^{(5)}\right.$.

TheOREM 7. If

1. $u(x, t) \in H, 0<t<c$,

2. $u(x, t) \geqq 0,0<t<c$,

3. $u(x, 0+)=0,-\infty<x<\infty$, then

$$
u(x, t)=0, \quad-\infty<x<\infty ; 0<t<c .
$$

For, by Theorem 6, we have from hypotheses 1 and 2 that

$$
u(x, t)=\int_{-\infty}^{\infty} k(y-x, t) d \alpha(y)
$$

where $\alpha(y)$ is non-decreasing. Let $y_{0}$ be any real number and let $\delta$ be any positive number. Then

$$
\begin{aligned}
u\left(y_{0}, t\right) & \geqq \int_{y_{0}}^{y_{0}+\delta} k\left(y-y_{0}, t\right) d\left[\alpha(y)-\alpha\left(y_{0}\right)\right] \\
& \geqq-\int_{y_{0}}^{y_{0}+\delta}(\partial / \partial y) k\left(y-y_{0}, t\right)\left[\alpha(y)-\alpha\left(y_{0}\right)\right] d y .
\end{aligned}
$$

If we perform the indicated differentiation and denote by $A$ a positive constant (which may change from one line to the next) we have

$$
\begin{aligned}
u\left(y_{0}, t\right) & \geqq(A / t) \int_{0}^{\delta} y k(y, t)\left[\alpha\left(y+y_{0}\right)-\alpha\left(y_{0}\right)\right] d y \\
& \geqq \underset{0 \leqq y \leqq \delta}{\text { g.l.b. }} \frac{\alpha\left(y+y_{0}\right)-\alpha\left(y_{0}\right)}{y} \frac{A}{t} \int_{0}^{\delta} y^{2} k(y, t) d y \\
& \geqq \underset{0 \leqq y \leqq \delta}{\text { g.l.b. }} \frac{\alpha\left(y+y_{0}\right)-\alpha\left(y_{0}\right)}{y} A \int_{0}^{\delta t^{-1 / 2}} y^{2} e^{-y^{2} / 4} d y .
\end{aligned}
$$

Now letting $t$ approach zero through positive values, we have

$$
0 \geqq \underset{0 \leqq y \leqq \delta}{\text { g.l.b. }} \frac{\alpha\left(y+y_{0}\right)-\alpha\left(y_{0}\right)}{y} A .
$$

(5) See, for example, G. Doetsch (1936). 
Since $\delta$ is arbitrary and since the right-hand side is certainly not negative, this becomes

$$
\liminf _{y \rightarrow 0+} \frac{\alpha\left(y+y_{0}\right)-\alpha\left(y_{0}\right)}{y}=0 .
$$

That is, the lower derivative on the right of $\alpha(y)$ is zero at $y_{0}$. The same must be true of the lower derivative on the left, by a similar argument. Then certainly $\alpha(y)$ is everywhere continuous. But a continuous function with any one of its four derivative numbers everywhere zero is necessarily constant. But if $\alpha(y)$ is constant, $u(x, t)$ is identically zero for positive $t$ by equation (12), and our theorem is completely established.

\section{BIBLIOGRAPHY}

G. Doetsch, Les équations aux derivées partielles du type parabolique, L'Enseignement Mathematique vol. 35 (1936).

J. B. J. Fourier, Theorie analytique de la chaleur, Paris, 1822.

E. Goursat, Cours d'analyse mathématique, Paris, 1923, vol. 3, chap. 29.

G. Herglotz, Über Potenzreihen mit positivem reelen Teil im Einheitskreis, Berichte über die Verhandlungen der königlich Sächsischen Gesellschaft der Wissenschaften zu Leipzig, mathematisch-physische Klasse vol. 63 [6-9] (1911) pp. 501-511. (1809).

P. S. Laplace, Memoire sur divers points d'analyse, J. Ecole Polytech. vol. 8, Cahier 15

L. H. Loomis and D. V. Widder, The Poisson integral representation of functions which are positive and harmonic in a half-plane, Duke Math. J. vol. 9 (1942).

S. D. Poisson, Theorie mathématique de la chaleur, Paris, 1835.

S. Täcklind, Sur les classes quasianalytiques des solutions des équations aux dérivées partielles $d u$ type parabolique. Doctor's thesis, Uppsala, 1936. Also Nova Acta Regiae Societatis Scientiarum Upsaliensis (4) vol. 10 (1936) pp. 1-57.

E. C. Titchmarsh, The theory of Fourier integrals, Oxford, 1937.

A. Tychonoff, Thêrèmes d'unicité pour l'équation de la chaleur, Rec. Math. (Mat. Sbornik) vol. 42 (1935).

$\mathrm{K}$. Weierstrass, Über die analytische Darstellbarkeit sogenannter willkürlicher Functionen reeller Argumente, Sitzungsberichte der Berliner Akademie, 1885.

D. V. Widder, The Laplace transform, Princeton, 1941.

HARVARD UNIVERSITY,

CAMBridge, Mass. 